\title{
Crowdfunding Social. La alternativa a la financiación tradicional de proyectos de organizaciones de participación
}

\author{
Natalia López Molero ${ }^{1}\left[\right.$; Elena Hernández Gómez ${ }^{2}$ y y Antonio Juan Briones Peñalver ${ }^{3}$
}

Recibido: 25 de noviembre de 2019 / Aceptado: 22 de mayo de 2020 / Publicado: 30 de octubre de 2020

Resumen. El crowdfunding es una alternativa a los métodos de financiación tradicional que pueden emplear las organizaciones de participación, mediante el uso de la comunidad como medio para la captación de fondos. Empleando la metodología de revisión de fuentes bibliográficas, nos adentraremos en el mundo del crowdfunding, estudiando las diversas interpretaciones del concepto, entre las que encontraremos al denominado crowdfunding social, sus modalidades de actuación, orígenes y plataformas donde se desarrolla la captación de fondos.

Así mismo, determinaremos los diferentes métodos de financiación que el emprendedor social puede emplear, para, posteriormente, realizar una comparación entre estos y el crowdfunding social, con el fin de conocer cuáles son las principales diferencias entre las variantes de búsqueda de financiación de proyectos, vista desde una óptica de beneficios e inconvenientes para el promotor del proyecto.

Palabras clave: Crowdfunding; Crowdfunding social; Organizaciones de participación; Financiación participativa; Plataformas de crowdfunding; Emprendedor social.

Claves Econlit: L31; O3; G21; G29.

\section{[en] Crowdfunding. The alternative to traditional financing of social projects}

\begin{abstract}
Crowdfunding stands as one of the main alternatives to traditional funding methods that participation organizations can use, using the community as a means of raising funds. Using the methodology of review of bibliographic sources, we will enter into the fascinating world of crowdfunding, studying the various interpretations of the concept, among which we will find the so-called social crowdfunding, its modalities of action, origins and platforms where the acquisition takes place funds.

Likewise, we will determine the different traditional financing methods that the social entrepreneur can use, to subsequently make a comparison between these and the crowdfunding, in order to know what the main differences are between these variants of seeking funding of projects, view from a perspective of benefits and disadvantages for the promoter of the project.
\end{abstract}

Keywords: Crowdfunding; Social crowdfunding; Participatory funding; Financing platform; Social entrepreneur.

Sumario. 1. Introducción. 2. Marco teórico de la investigación. 3. Metodología empleada. 4. Resultados de la comparativa entre crowdfunding y fuentes de financiación tradicionales. 5. Conclusiones, limitaciones y futuras líneas de investigación. 6. Referencias bibliográficas.

Cómo citar. López Molero, N.; Hernández Gómez, E.; Briones Peñalver, A.J. (2020) Crowdfunding Social. La alternativa a la financiación tradicional de proyectos de organizaciones de participación. REVESCO. Revista de Estudios Cooperativos, vol. 136, e71851. https://dx.doi.org/10.5209/reve.71851.

\section{Introducción}

La financiación de las empresas y las entidades sociales, se encuentra inmersas en una etapa de profunda transformación, derivada de los numerosos avances en materia tecnológica y el uso de internet para el desarrollo de todo tipo de actividades empresariales. Además, la crisis financiera de 2008, afectó considerablemente a estas entidades, dado que, con la escasez de fondos, muchas pymes, grandes empresas, entidades de la economía social, así como, aquellos proyectos de carácter emprendedor, con fines sociales, se

1 Universidad Politécnica de Cartagena, España.

Dirección de correo electrónico: natalia.lmolero@edu.upct.es.

2 Universidad Politécnica de Cartagena, España.

Dirección de correo electrónico: elena.hernandez@upct.es.

3 Universidad Politécnica de Cartagena, España.

Dirección de correo electrónico: Aj.briones@upct.es. 
vieron en la dificultad de obtener financiación para desarrollar su actividad. Por último, cabe destacar, que, aunque todavía no hay datos fiables para poder cuantificar las consecuencias económicas, financieras, así como sociales, diremos que la actual situación de emergencia sanitaria global en el año 2020 resultante de la pandemia del COVID-19, va a afectar a la financiación de estas entidades de una manera bastante relevante y significativa.

Dicho esto, las Entidades de la Economía Social deben recurrir a otro tipo de alternativas financieras, como puede ser el crowdfunding, para solucionar esta falta de fondos. Por este motivo, este problema afecta de lleno al Tercer Sector, y concretamente, a entidades que participan en mercado de la economía social.

Utilizando como base el artículo "Análisis de las plataformas de crowdfunding social en el estado español: un estudio de casos", elaborado por Antonia Sajardo (2018), procederemos a analizar el potencial del crowdfunding, así como, del crowdfunding social como alternativa a las fuentes de financiación tradicionales, utilizadas para financiar proyectos de organizaciones de participación del Tercer Sector de Acción Social en España, justificando el papel que desempeñan estas plataformas en la financiación de proyectos sociales, mediante el análisis de las ventajas e inconvenientes que presenta el crowdfunding con respecto a otras fuentes tradicionales. Además, hemos revisado autores como Howe (2008), Belleflamme (2012), Steinberg y DeMaria (2012), Gallardo (2015), entre otros, que son expertos en esta materia, lo cual nos ha permitido construir un marco teórico sólido para el trabajo que presentamos.

Esta investigación se desarrollará en tres grandes etapas. En la primera etapa estableceremos, mediante la aplicación de una metodología basada en la revisión de fuentes bibliográficas, un marco teórico en torno a los conceptos de: crowdfunding, crowdfunding social, fuentes de financiación tradicionales a disposición del emprendimiento social y organizaciones de participación social.

En la segunda etapa del trabajo, procederemos a exponer las ventajas e inconvenientes tanto del crowdfunding como de las fuentes tradicionales, vistas, primeramente de manera generalizada y posteriormente individualizada, mediante la elaboración de una tabla comparativa con cuatro variables de comparación: tipo de inversor que opera, fase del proyecto en la que se especializa, tipo de contraprestación que el promotor tiene que ofrecer al inversor y nivel de requisitos para acceder a la financiación.

Finalmente, en la etapa final, procederemos a exponer las conclusiones de esta investigación, así como, las limitaciones surgidas en el desarrollo del mismo y las futuras líneas de investigación.

\section{Marco teórico de la investigación}

\subsection{Crowdfunding}

A pesar de que la primera mención escrita de este concepto es relativamente reciente, en concreto, realizada por Michael Sullivan en 2006, podemos decir que este concepto se puede entenderse como "parte del amplio concepto de crowdsourcing, que se refiere al uso de la «(multitud») para la obtención de ideas, información y soluciones para el desarrollo de actividades empresariales" (Schwienbacher, Belleflamme y Lambert, 2012). Por tanto, identificaremos como origen del crowdfunding al denominado crowdsourcing.

El uso de la multitud como medio para la obtención de información, ideas, sugerencias, soluciones para problemas empresariales se ha dado a lo largo del tiempo ${ }^{4}$, pero solo a finales del siglo XX, este crowdsourcing se identificó con la externalización que realiza una empresa o institución de algunas de las funciones que realiza un empleado a un grupo indefinido de personas mediante una convocatoria abierta (Howe, 2006). Este término, al igual que el crowdfunding, ha sido sujeto a múltiples interpretaciones de distintos autores (véase figura 1).

Consideramos a Demócrito, filósofo griego 480-370 a.C., como la primera personas en establecer la necesidad que tiene la especie humana de organizarse en grupos de personas con intereses comunes, como medio para desarrollarse. Otro caso destacado es el experimento que presenció Francis Galton, en la obra The Wisdom of Crowds, donde pudo ver como un grupo de personas, que presenciaban una feria de ganado, sin concimientos previos sobre ganaderia, se aproximaban al peso exacto de una cabeza de ganado. 
Figura. 1. Evolución del concepto de crowdsourcing.

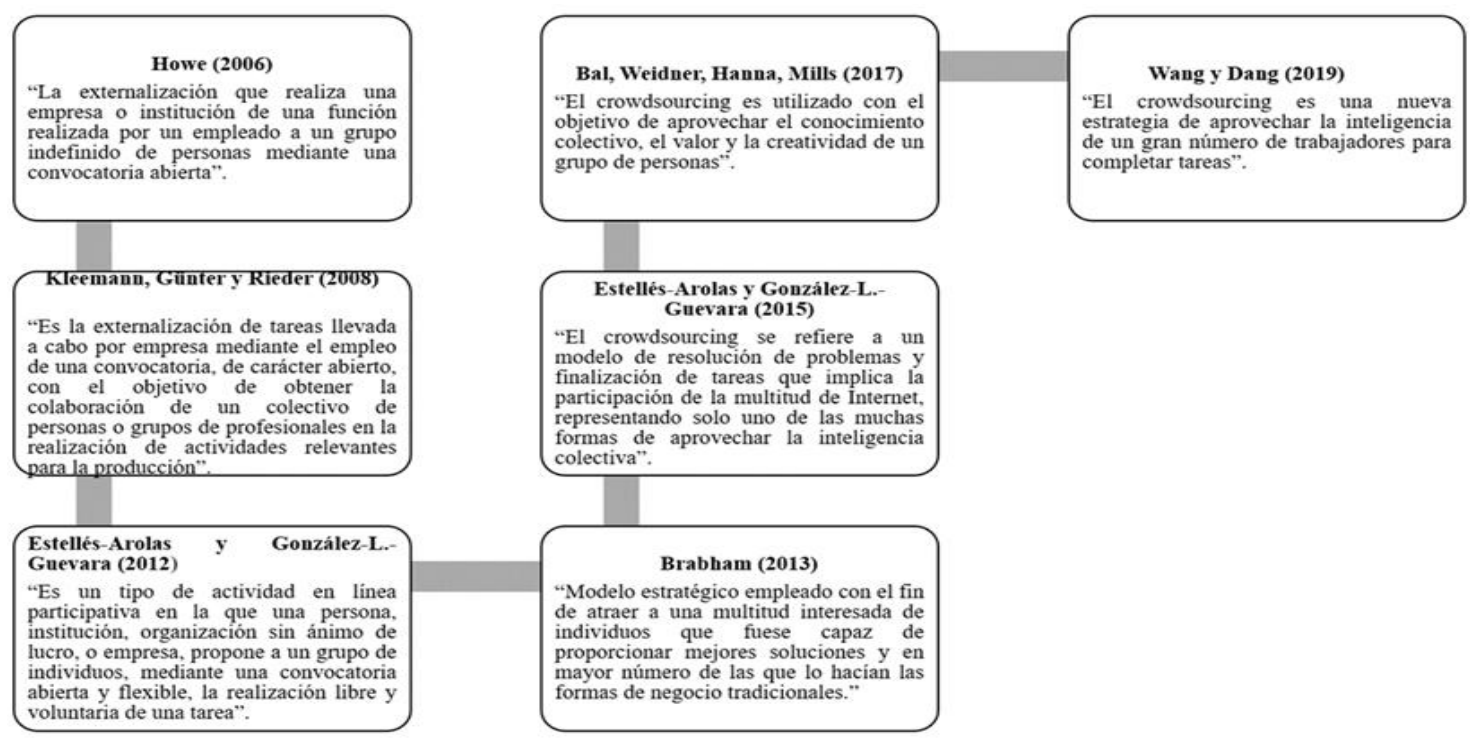

Fuente:Elaboración propia.

"Financiación colectiva", "financiación en masa", "micro mecenazgo", "micro financiación colectiva" .... son algunas de las múltiples traducciones del vocablo inglés crowdfunding, siendo este considerado como un "sistema de financiación colectiva mediante el cual muchos proyectos pueden ser reales gracias a las aportaciones que realizan inversores particulares, destacando el importantísimo papel que juega internet y las redes sociales que constituyen la base del funcionamiento de las diversas plataformas que operan en nuestro país" (Gallardo, 2015).

A pesar de ser un término relativamente reciente, dado que, su nacimiento se sitúa en 2006, multitud de autores se han prestado a dar una definición (veáse figura 2).

Figura. 2. Evolución del concepto de crowdfunding.
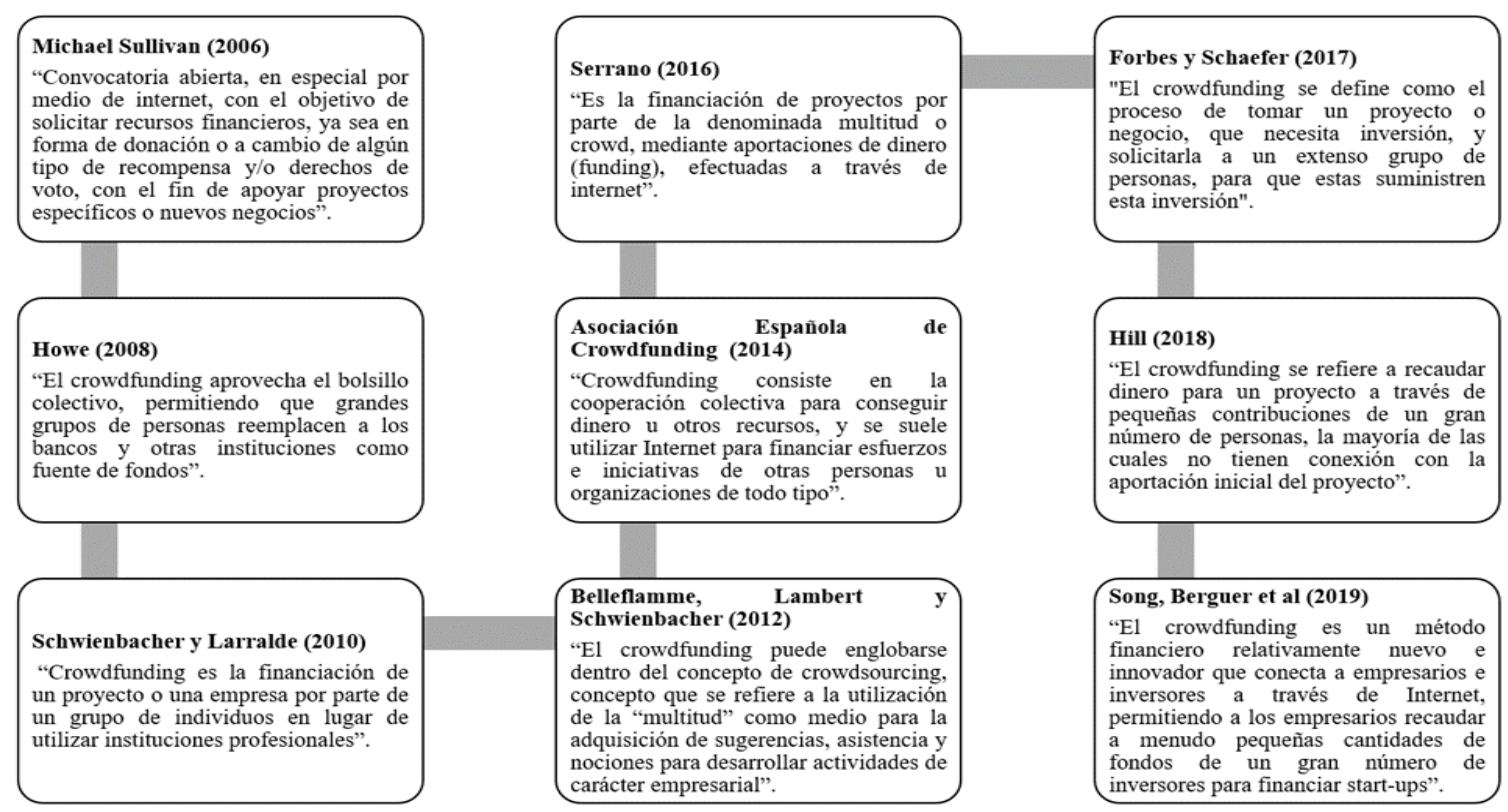

Fuente: Elaboración propia.

En resumen, diremos que el crowdfunding implica la búsqueda de financiación por parte de personas/empresas/entidades con el objetivo de poner en marcha sus proyectos, utilizando para ello aportaciones de dinero realizadas por personas interesadas, siendo imprescindible internet como medio en el cual lanzar el proyecto en las denominadas plataformas de crowdfunding que funcionaran como canales de 
comunicación entre el promotor y el inversor. Es considerado como la máxima expresión, en términos de financiación, del crowdsourcing y se erige como una de las principales alternativas a la financiación bancaria.

Tomando como base a Steinberg y DeMaria (2012), Gallardo (2015), Sajardo (2016) y Olarte-Pascual, Sierra-Murillo y Ortega (2016), procedemos a definir cuáles son los actores participantes, así como, los modelos de funcionamiento que emplean las plataformas de crowdfunding. En relación a los actores participantes, en el desarrollo de un proyecto de crowdfunding, son, como mínimo, tres (véase figura 3).

Figura. 3. Integrantes en un proyecto de crowdfunding.

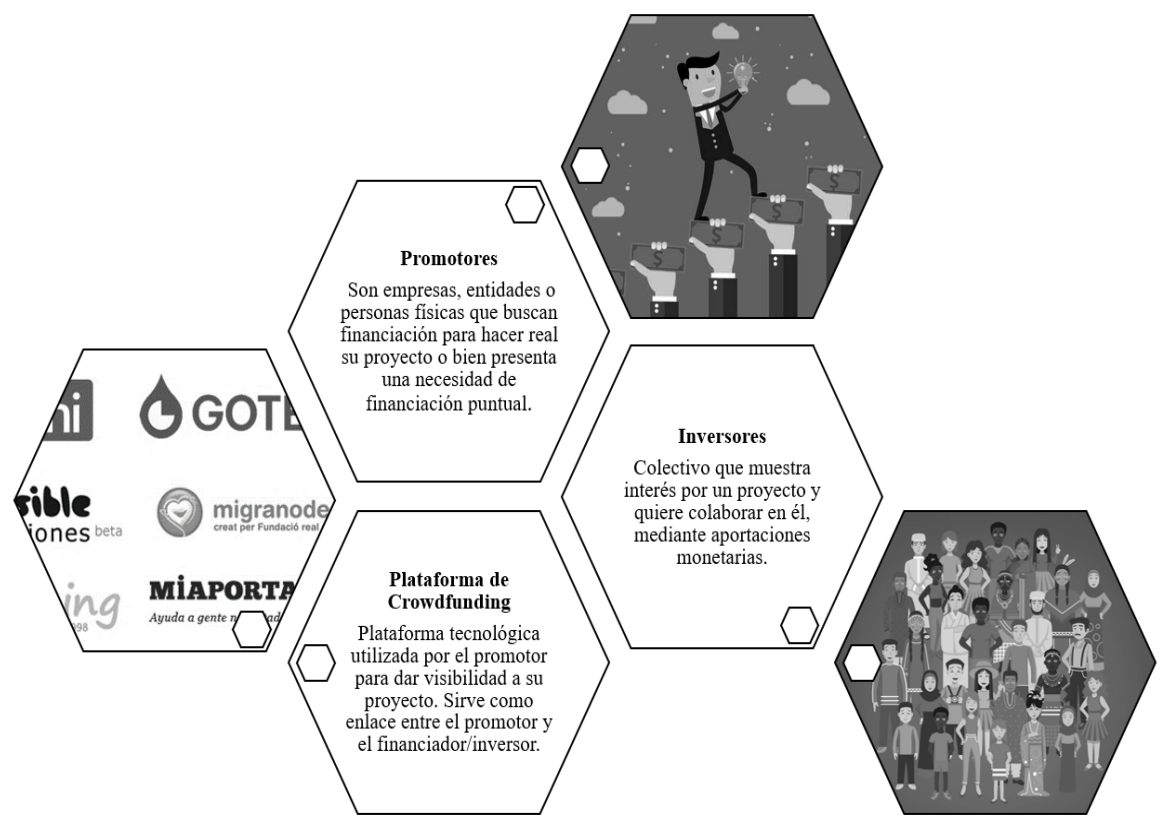

Fuente: Elaboración propia.

En cuanto al funcionamiento de las plataformas de crowdfunding diremos que, estas funcionan bajo dos grandes modelos:

- Modelo "Todo o nada": Las plataformas que funcionen bajo este modelo, indican al promotor que como condición para poder disponer de los fondos, tienen que cumplir con el objetivo de financiación que este haya establecido. En caso de incumplimiento del objetivo, la plataforma devolverá a cada inversor que participó, la cantidad que este aportó al proyecto.

Modelo "Todo suma": Las plataformas que funcionen bajo este modelo, establecen que los promotores de los proyectos publicados, puedan disponer de todos lo fondos que hayan captado, aunque no se haya llegado al objetivo de financiación establecido.

En relación a las diversas modalidades de crowdfunding existentes, vamos a realizar su clasificación atendiendo a la contraprestación que el promotor realiza a los aportantes de los fondos: 
Figura. 4. Modalidades de crowdfunding

\begin{tabular}{|c|}
\hline Donación (Donation-based crowdfunding) \\
\hline $\begin{array}{l}\text { Esta modalidad está caracterizada por que los aportantes de los fondos no } \\
\text { reciben ningún tipo de contraprestación, destacando, el marcado carácter } \\
\text { social y humanitario que tienen los proyectos que buscan financiación } \\
\text { empleando esta modalidad. } \\
\text { Los promotores suelen funcionar bajo la fórmula de ONG o asociaciones } \\
\text { que operan en el sector no lucrativo y buscan que los proyectos sean } \\
\text { considerados como una muestra de solidaridad. }\end{array}$ \\
\hline Recompensa (Lending-based crowdfunding) \\
\hline $\begin{array}{l}\text { Esta modalidad está caracterizada por que los aportantes de capital reciben } \\
\text { una contraprestación correspondiente a la totalidad del dinero aportado más } \\
\text { unos intereses pactados entre el promotor y el aportante. } \\
\text { En esta modalidad, el aportante busca una rentabilidad al capital que aporta, } \\
\text { siendo esta rentabilidad la resultante de la suma del capital devuelto más } \\
\text { unos intereses, que como hemos dicho anteriormente son pactados ente el } \\
\text { promotory el financiador. }\end{array}$ \\
\hline Préstamo (Reward crowdfunding) \\
\hline $\begin{array}{l}\text { Esta modalidad es una de las más extendidas por todo el mundo, estando } \\
\text { destinada sobre todo a las empresas de nueva creación, funcionando como } \\
\text { un intercambio entre el promotor y el financiador, comprometiéndose el } \\
\text { primero a entregar un producto o servicio que tenga pensado ofrecer una } \\
\text { vez que la empresa se encuentre en funcionamiento a modo de } \\
\text { contraprestación. } \\
\text { Esta modalidad está muy extendida para proyectos de carácter artístico o } \\
\text { cultural. El funcionamiento de las plataformas que operan bajo esta } \\
\text { modalidad se rige por el modelo "todo o nada". }\end{array}$ \\
\hline Inversión (Equity crowdfunding) \\
\hline $\begin{array}{l}\text { Esta modalidad consiste en que el financiador aporta dinero a la } \\
\text { organización a cambio de que esta le facilite una contraprestación que toma } \\
\text { la forma de acciones o participaciones en el negocio, de manera que, este } \\
\text { financiador se convierte en inversor y participante de la organización, } \\
\text { aunque en ocasiones se puede dar el caso de que la participación sea solo } \\
\text { sobre el beneficio de la empresa sin la posibilidad de voto. } \\
\text { Esta modalidad tiene como característica definitoria que debe transcurrir un } \\
\text { período de tiempo superior a las modalidades anteriores, con el fin de que } \\
\text { la empresa adquiera un determinado volumen de fondos que le permita } \\
\text { hacer frente a las contraprestaciones. }\end{array}$ \\
\hline
\end{tabular}

Fuente: Elaboración propia.

Por último y para finalizar con este apartado, diremos que la Ley 5/2015 del 27 de abril, de fomento de la financiación empresarial, define a las plataformas de crowdfunding como "empresas autorizadas cuya actividad consiste en poner en contacto, de manera profesional y a través de páginas web u otros medios electrónicos, a una pluralidad de personas físicas o jurídicas que ofrecen financiación a cambio de un rendimiento dinerario, denominados inversores, con personas físicas o jurídicas que solicitan financiación en nombre propio para destinarlo a un proyecto de financiación participativa, denominados promotores", pudiendo funcionar en múltiples sectores de actividad, desde el apoyo a la creación de empresas y startups hasta la financiación de actividades deportivas, culturales e incluso están presentes en la financiación de proyectos relacionados con la salud y ciencia.

Según el último informe anual sobre la Financiación Participativa en España, elaborado por Universo Crowdfunding en colaboración con la Universidad Politécnica de Madrid, se establece que hay activas, en 2018, 50 plataformas que operan en distintos sectores de la economía española, así como dedicadas a las distintas modalidades existentes de crowdfunding (véase figura 4): 
Figura. 5. Diversas plataformas de crowdfunding en función de la naturaleza del proyecto.
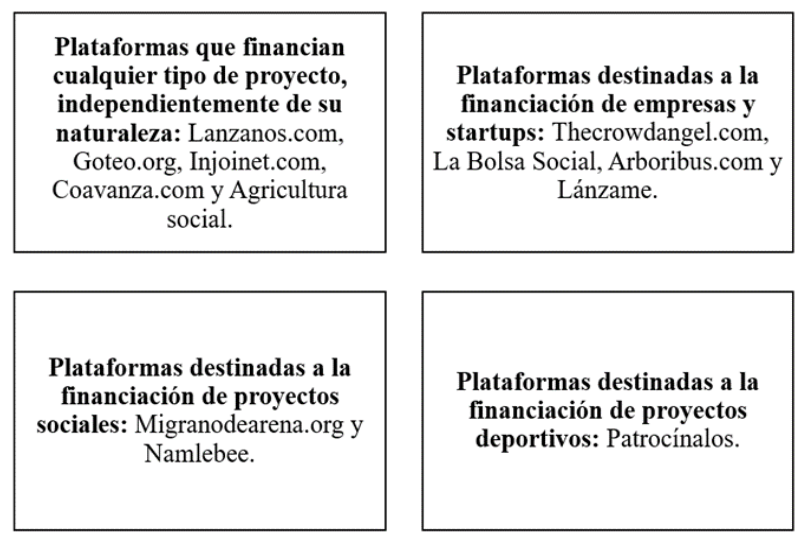

Fuente: Elaboración propia.

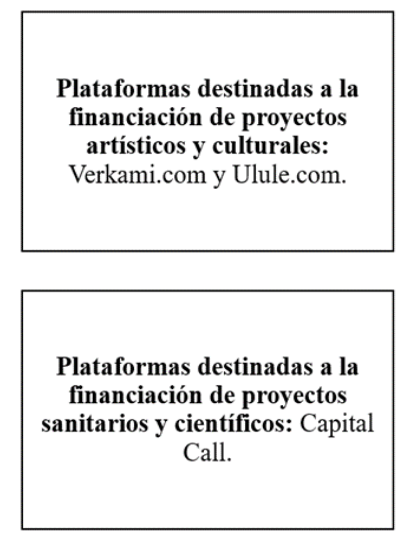

Plataformas destinadas a la financiación de proyectos Call.

\subsection{Crowdfunding social}

Este estudio, como ya se expuso en la introducción, tiene como base la investigación de Sajardo (2018), "Análisis de las plataformas de crowdfunding social en el estado español: un estudio de casos". Tal y como expone Sajardo (2018), el estudio del crowdfunding como herramienta financiera de gran utilidad para las entidades sociales, ha sido recientemente realizado en el ámbito de maneral parcial.

Pese a esto, podemos equiparar el crowdfunding social con el crowdfunding de donaciones, dado que, ambas modalidades se reservan para la financiación de proyectos de corte solidario y que buscan generar un impacto positivo en la sociedad, o, bien, crear valor social. Este valor social, tal y como recoge Lejarriaga y Bel (2018), puede manifestarse de diversas formas como pueden ser las siguientes: respeto al medioambiente, favorecer la inclusión social, cohesión social, etc.

Esto implica que los inversores aportan fondos bajo esta modalidad, lo hacen, no con el objetivo de percibir una contraprestación, dado que, en este caso no hay una contraprestación tangible; sino que buscan el desarrollo del proyecto por su interés en él. El tipo de proyectos que tienen cabida en este tipo de crowdfunding van desde proyectos de naturaleza sanitaria, promovidos por ONG, fundaciones, asociaciones sin ánimo de lucro a proyectos de mejora del impacto medioambiental en determinados procesos (Sajardo, 2012).

Por tanto, y bajo la premisa anterior, podemos decir que el crowdfunding social, puede tener una especial utilidad en la captación de fondos en proyectos de corte más solidario y cuyos inversores busquen como recompensa un reconocimiento intangible . $^{5}$.

El funcionamiento de este tipo de crowdfunding es el siguiente (Sajardo, 2017:133):

1. "La entidad no lucrativa (promotor) confecciona la idea o proyecto a financiar, es decir, describe su objetivo, indicando la cantidad necesaria para llevarlo a cabo, el tiempo de recaudación establecido y el plan de recompensas que se quiere ofrecer a cada persona que aporte dinero según la cantidad que decida donar".

2. "El proyecto se implementa en una plataforma web, proveedora de servicios de crowdfunding, que validará y hará pública la campaña correspondiente mediante la utilización de estrategias propias".

En la actual situación de emergencia sanitaria, este tipo de crowdfunding resulta fundamental para prestar apoyo a aquellos colectivos que se encuentran más afectados. Un ejemplo de esta utilidad, puede ser los proyectos impulsados por la plataforma migranodearena.org, bajo el nombre \#coronaretos (https://www.migranodearena.org/eventos/319/coronaretos), donde llevan recaudados más de millón de euros destinados a la financiación de diversos planes de apoyo afectados por el COVID-19, entre los que destacaremos el proyecto impulsado por Cáritas Diocesana de Cádiz, que busca conseguir fondos para la ayuda a aquellas familias en riesgo de exclusión social, que resultan afectadas por el cierre de negocios provocados por la declaración del estado de alarma en España durante el año 2020, derivada de la pandemia de COVID-19.

Sajardo Moreno, A., Gil Ibáñez, M., \& Pérez Sempere, S. (2017). El sector no lucrativo en el marco de la nueva era tecnológica: el desafío del Crowdfunding social en España. Análisis del caso de la asociación Alba Pérez, lucha contra el cáncer infantil. REVESCO. Revista De Estudios Cooperativos, 124, 128-151. 


\subsection{Organizaciones de participación social}

Consideramos la participación como el acto mediante el cual nos involucramos en una organización, con el fin de conseguir una mejora en la productividad y competitividad, no solo de la organización en sí, sino también a título personal. Esta participación, será mayor en las épocas de crisis en la empresa, ya que, no solo buscaremos la supervivencia de la organización, sino también nuestra propia supervivencia.

Las organizaciones de participación pueden ser consideradas como "entidades que se rigen con los principios de democracia y solidaridad, recibiendo recursos financieros de los poderes públicos o, bien, están subvencionadas bajo la hipótesis de que si el poder público correspondiente asumiera la función que realizan estas entidades resultaría más costoso. Sin embargo, son autónomas, aunque no funcionan con los criterios de competitividad que establece el mercado" (García-Gutiérrez, 2002:102-106).

En el ámbito más concreto de la economía social, diremos que las organizaciones de participación son "aquellas organizaciones donde las personas tienen la capacidad de participar en la fijación de los objetivos de estas" (García- Gutiérrez, 2002:106), siendo necesario ser o bien proveedor o bien consumidor de bienes y servicios, o ambas cosas, para poder participar.

Estas organizaciones de participación pueden ser clasificadas atendiendo a si estas producen y distribuyen bienes y servicios (García-Gutiérrez, 2002):

Figura. 6. Tipos de organizaciones de participación.

\begin{tabular}{|c|c|}
\hline Organizaciones empresariales "de participación" & $\begin{array}{c}\text { Organizaciones no empresariales "de } \\
\text { participación" }\end{array}$ \\
\hline $\begin{array}{l}\text { - Son organizaciones en la que lo que confiere poder } \\
\text { de decisión para establecer los objetivos de la } \\
\text { empresa, es la participación, como protagonistas, } \\
\text { promotores, actores, en el proceso de producción- } \\
\text { distribución de bienes y servicios (García-Gutiérrez, } \\
\text { 2002). En estas entidades se antepone las personas y } \\
\text { sus intereses sobre el capital. } \\
\text { - Son: Cooperativas de primer grado, mutuas y } \\
\text { mutualidades, cofradías, sociedades laborales, } \\
\text { sociedades agrarias de transformación y cajas de } \\
\text { ahorro. }\end{array}$ & $\begin{array}{l}\text { - Son entidades que pueden producir bienes y } \\
\text { servicios, pero cuya distribución se realiza al } \\
\text { margen del mercado, además, de realizar actividades } \\
\text { encaminadas a atender aquellas necesidades sociales } \\
\text { más "abandonadas" por la administración pública. } \\
\text { Estas entidades pueden surgir de iniciativas de la } \\
\text { sociedad, grupos religiosos o por grupos políticos. } \\
\text { - Son: Asociaciones, fundaciones, sindicatos, } \\
\text { voluntariado, las empresas sin fin de lucro y } \\
\text { organizaciones no gubernamentales. }\end{array}$ \\
\hline
\end{tabular}

Fuente: Elaboración propia a partir de García- Gutiérrez (2002).

\subsection{Fuentes de financiación tradicionales del emprendimiento social}

Sánchez, Martín, Durán y Lejarriaga (2018:20), definen el emprendimiento social como "aquella iniciativa que a través de fórmulas de mercado o no mercado contribuye a la cohesión social basándose en la primacía de las personas sobre el capital y con gobernanza democrática y/o participativa”.

Otros autores, además, añaden que es una combinación entre el ingenio del espíritu tradicional con la misión de cambiar la sociedad (Seelos y Mair, 2005, p. 241), abarca actividades y procesos realizados para descubrir, definir y aprovechar las oportunidades con el objeto de mejorar la riqueza social (Zahra et al., 2009, p. 519) siendo llevada a cabo por organizaciones sin ánimo de lucro, empresas u organismos gubernamentales (Guzmán y Trujillo, 2008, p. 111).

Tras esta explicación, procederemos a ver cuales son las fuentes más relevantes a disposición del emprendedor (Messina y Pena, 2013: 27):

\subsubsection{Friends, Family and fools}

También denominada las 3F de la financiación. Esta fuente consiste en utilizar de los recursos financieros del entorno cercano al emprendedor, es decir, el emprendedor conseguirá financiación para su proyecto solicitando financiación a sus familiares y personas cercanas a su entorno, jugando un papel fundamental el denominado "vínculo emocional" que tenga el emprendedor con su entorno, ya que cuanto mayor sea este vínculo, más facilidad tendrá el emprendedor de convencer a los inversores de invertir en el proyecto. Es una fuente perfecta para las etapas tempranas del proyecto, siendo en algunos casos motivo por el cual hay proyectos que se desarrollan y otros que no. 


\subsubsection{Préstamo bancario}

Es una operación, de naturaleza financiera, estipulada mediante un contrato, donde se establece que la entidad financiera pone a disposición del emprendedor una cierta cantidad de dinero, que este tiene que devolver en un plazo de tiempo establecido en el contrato, con una contraprestación pactada, en forma de tipo de interés.

Para poder acceder a ella, es necesario que el emprendedor presente una serie de garantías a la entidad financiera a fin de reducir el riesgo de la operación, siendo estas tanto personales, como asociadas al valor de un bien inmueble. El emprendedor deberá pagar cuotas, que pueden ser mensuales, trimestrales o anuales, o bien se establecerá de mutuo acuerdo con la entidad financiera.

Esta fuente presenta una serie de modalidades diferentes: préstamos personales, préstamos hipotecarios o préstamos pignoraticios. Y para finalizar el emprendedor incurre en otra serie de costes (comisión de apertura, comisión de estudio, comisión de cancelación, entre otros gastos), distintos al tipo de interés.

\subsubsection{Líneas de crédito}

La entidad bancaria facilita al emprendedor un importe monetario pactado con anterioridad, del que el emprendedor podrá disponer ya sea total o parcialmente, con el fin de cubrir las necesidades de efectivo que el emprendedor presenta durante un periodo de tiempo determinado, generalmente igual o menor a un año. La garantía que la entidad financiera reclama al emprendedor, suele ser de naturaleza personal.

Presenta unos gastos similares a los préstamos, resaltando que, normalmente, el tipo de interés, que paga el emprendedor, es superior a otros productos de financiación.

Esta fuente, aunque a primera vista puede ser muy similar a un préstamo bancario, presenta una serie de diferencias con respecto a este:

- $\quad$ El tipo de interés suele ser superior que los tipos de interés en los préstamos.

- El plazo de amortización de las líneas de crédito es menor que en los préstamos, por ejemplo, los préstamos tienen un periodo de amortización de entre 5 a 40 años, dependiendo de la naturaleza del mismo, mientras que las líneas de crédito no suelen tener un plazo superior al año.

- A diferencia del préstamo, en la línea de crédito no hay cuotas mensuales, sino que al final del plazo de amortización el emprendedor devolverá el capital.

\subsubsection{Microcréditos}

Es un producto bancario destinado a colectivos que presentan dificultades para acceder a financiación, ya sea por su situación sociolaboral, como por la ausencia de avales o garantías que les permitan acceder a un préstamo o crédito.

Estos microcréditos están dentro del denominado grupo de productos bancarios sociales, es decir, aquellos productos bancarios cuyo fin último no es solo la generación de empleo, sino también la consecución de un beneficio social y no lo ofrecen las entidades financieras, sino tambien fundaciones y asociaciones.

Los colectivos que utilizan esta fuente de financiación suelen usarla para iniciar y desarrollar proyectos cuyo fin es la generación de empleo o la creación de microempresas.

Generalmente, los microcréditos no suponen para el emprendedor la generación de gastos diferentes a la devolución del capital prestado y el tipo de interés que soportan es inferior al tipo de interés del mercado.

\subsubsection{Ayudas y subvenciones públicas}

Son consideradas como "contribuciones financieras o transferencias de fondos procedentes de un gobierno u organismo público, estructurada mediante un programa público de subvenciones y que tiene como destino dotar de fondos al sector privado, en este caso las empresas, sin una devolución posterior del capital obtenido o a un coste muy bajo en comparación con otras alternativas de financiación" (Rodríguez-Carmona et al., 2008). Además, pueden englobarse dentro de los programas promovidos por la administración pública, cuyo fin es el fomento de determinadas actividades o, cuyo destino, está reservado a determinados colectivos.

Tienen carácter público, encontrándose controlada por organismos y entidades que pueden transferir esta administración a entidades, de carácter local, instituciones financieras, fundaciones y asociaciones. Las cuantías de fondos transferidos u otorgados al emprendedor, generalmente, no son objeto de devolución, o bien, esta devolución supone un coste muy reducido. 


\subsubsection{Business Angels}

Son considerados inversores que presentan numerosos conocimientos, de naturaleza técnica, en áreas diversas, así como, una alta capacidad económica, que podrán a disposición del emprendedor de un proyecto, caracterizado por tener un amplio potencial de crecimiento, con el fin de que este se desarrolle, por tanto, esta fuente no solo aporta financiación, sino también, conocimientos y una red de contactos, ya que el objetivo de los inversores es que el proyecto crezca para poder obtener su contraprestación. A cambio, el inversor requerirá, que el emprendedor, le ofrezca una rentabilidad a medio plazo.

Estos inversores suelen ser personas físicas, que poseen amplios conocimientos y experiencia en los sectores donde van a invertir. Su capacidad de inversión se sitúa entre los 25.000 a 250.000 euros y no siempre tienen como prioridad la rentabilidad del proyecto. En ocasiones, el motivo de su inversión se encuentra en factores tales como: satisfacción profesional, vínculos afectivos, etc.

Pueden agruparse para formar una red de business angels, canal que permite la comunicación entre empresas y emprendedores que busquen captar fondos para sus proyectos. Es interesante resaltar, que estas redes poseen una estructura organizativa, encargada de estudiar los proyectos que presenten una necesidad de financiación y buscarles el inversor más adecuado. Desde la óptica del inversor, la red facilitara información sobre el proyecto.

Este un tipo de financiación, más específica para proyectos iniciados por emprendedores en sus primeras fases de desarrollo.

\section{Metodología empleada}

Esta investigación se realiza con el objetivo de responder a tres preguntas esenciales:

1. ¿Qué es el crowdfunding y como este puede ser empleado como fuente opcional a fuentes de financiación tradicionales para proyectos de carácter social?

2. ¿Cuáles son las fuentes de financiacion tradicionales más conocidas que pueden emplearse para la financacion de proyectos de carácter social?

3. ¿Cuáles son las principales ventajas e inconvenientes de este crowdfunding y en que se difieren con respecto a las fuentes tradicionales?

Para ello desarrollaremos una metodología cualitativa basada en la revisión de fuentes bibliográficas de distinta naturaleza, como son las bases de datos donde buscaremos, principalmente, artículos de investigación y académicos, libros y revistas que tratan la temática de la investigación, y legislación vigente en España relacionada con este tema ., como es la Ley 5/2015 de fomento de la financiación empresarial.

Las bases de datos empleadas son Google Scholar y ScienceDirect, a las cuáles hemos aplicado los siguientes comandos de búsqueda, con el fin de delimitar el volumen de información:

- «Crowdfunding».

- $\quad$ «Crowdsourcing».

- $\quad$ «Emprendimiento».

- $\quad\langle$ Emprendimiento social».

- $\quad$ «Fuentes financiación tradicionales emprendimiento».

- «Crowdfunding social».

- «Financiación participativa».

- «Organizaciones de participación social».

A pesar de esta delimitación la información disponible es muy extensa abarcado, aproximadamente, más de 171.000 referencias. A fin de emplear la información más relevante y útil, procedimos a emplear diversos criterios de selección:

Fecha de publicación: 2006 a 2019.

- Tipo de artículo: academico, investigacon o revision bibliografica.

- Autor6: Se da preferencia a artículos de autores destacados como Howe, Bellenflamme, Sajardo, Estelle-Arollas, entre otros.

Estas delimitación, nos permitieron seleccionar 28 artículos esenciales para nuestra investigación. Además de la documentación proveniente de las bases de datos anteriormente mencionadas, se ha empleado una serie de libros y artículos de revistas relacionadas con la economía social:

6 Esta preferencia está establecida en función del número de artículos que estos autores han publicado sobre las materias que tratamos en el presente artículo. 
1. Libros:

- The Crowdfunding Bible: How to raise money for any startup, video game, or Project. Elaborado por Scott Steinberg.

- ¿Qué es y cómo gestionar el crowdfunding? Elaborado por Eva Gallardo, Joan Torres y M. Consol Torreguitart Miranda.

- Crowdfunding: Aspectos Legales. Elaborado por Enrique Moreno et al.

2. Revistas especializadas en economía social:

- Oikonomics.

- REVESCO.

- CIRIEC.

Por último, y para concluir con la metodología, diremos que hemos llevado a cabo una revisión de toda la normativa relacionada con el crowdfunding vigente, en territorio español.

Figura. 7. Metodología aplicada en la investigación.

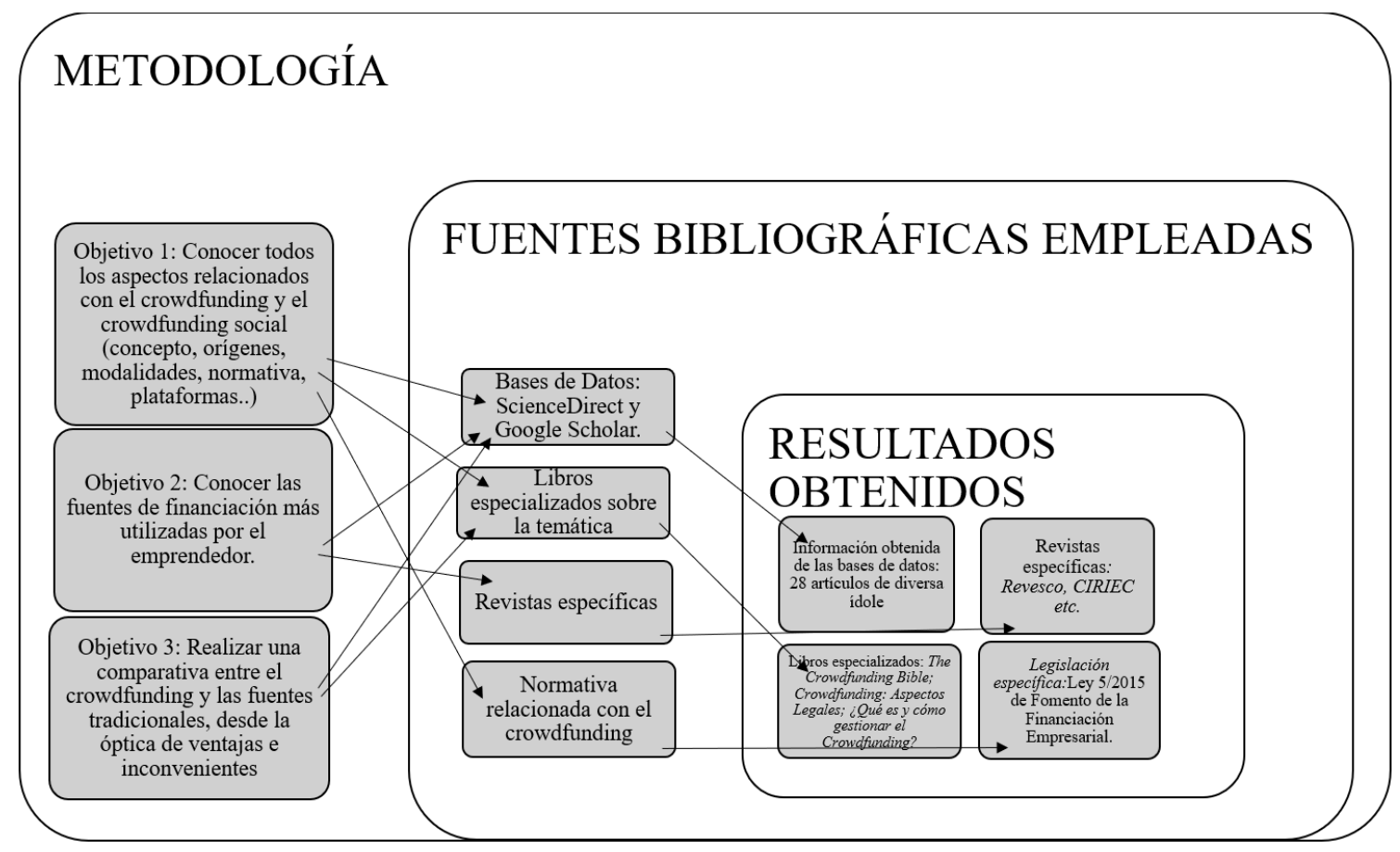

Fuente:Elaboración propia.

\section{Resultados de la comparativa entre crowdfunding y fuentes de financiación tradicionales}

\subsection{Crowdfunding. Ventajas e inconvenientes}

Como ya hemos visto con anterioridad, el crowdfunding es un método de financiación, basado en la utilización de la comunidad, como medio de obtención de fondos para el avance de sus proyectos, siendo imprescindible el uso de internet como medio donde desarrollar todo el proceso de captación de fondos.

La utilización de esta fuente de financiación otorga una serie de ventajas a los promotores de un determinado proyecto, que identificaremos como el emprendedor:

- $\quad$ El promotor tiene el control sobre la totalidad de los aspectos inherentes de su proyecto (visión creativa, estrategias a aplicar, costes etc.).

- $\quad$ El promotor determina el tipo de relación que establecerá con sus clientes potenciales.

- El promotor, cuando publica su proyecto en una plataforma, es consciente de que debe aplicar estrategias captación de nuevos inversores, así como, técnicas de marketing para hacer su proyecto más atractivo de cara a sus inversores, ya que estos pueden convertirse en clientes potenciales. 
En las situaciones donde el promotor no consigue la financiación necesaria para el proyecto, la pérdida que se produce es de tiempo y un daño ocasional en la imagen del promotor de fácil solución: con que el promotor realice una mejora de la imagen y redifinición del plan que sustenta el proyecto, el promotor recuperará su prestigio anterior a la búsqueda de financiación.

Para aquellos promotores y entidades que pertenecen al sector no lucrativo, este tipo de financiación es ideal, dado que, reduce la dependencia que estas puedan tener de sus donantes habituales, facilitando la obtención de fondos sin ser necesario hacer uso de las fuentes de financiación tradicionales.

En el caso de que el promotor obtenga una cantidad superior a la estimada, no tiene la obligación de justificarla frente a ningún tipo de organismo de control.

- $\quad$ Esta fuente ofrece, además, el beneficio inesperado de que el promotor puede recibir ofertas, no sólo de tipo monetario, sino también de asistencia de aquellos inversores que muestran interés en el avance del proyecto.

Pero esta fuente no sólo otorga ventajas al promotor, sino que también, puede presentar una serie de inconvenientes:

- El desarrollo de un proceso de crowdfunding puede suponer un factor de estrés para el promotor, dado que, tiene que dirigir un proyecto que resulte atractivo a la comunidad de donde surgirán sus inversores, mediante el establecimiento de una campaña de atracción, que supone un esfuerzo considerable para el promotor.

El promotor del proyecto, tiene que realizar grandes esfuerzos en materia de autopromoción y campañas de marketing, de manera constante, ya que sin estas dos acciones el éxito del proceso de crowdfunding no se va a dar.

El promotor debe asumir la responsabilidad de tratar con el conjunto de inversores o patrocinadores del proyecto, siendo estos un grupo con expectativas y demandas heterogéneas.

- $\quad$ El promotor debe realizar una búsqueda constante de nuevas formas de publicidad, a fin de que se mantenga y aumente el interés que tenga la sociedad sobre el proyecto.

Es un proceso que, aunque en la mayoría de casos finaliza con éxito, está sujeto a situaciones que suponen altibajos para el ánimo del promotor, derivando en las situaciones de estrés anteriormente mencionadas.

\subsection{Fuentes de financiación tradicionales. Ventajas e inconvenientes.}

Como aparece expuesto en el marco teórico, las fuentes de financiación tradicionales que puede emplear el promotor de un determinado proyecto son las siguientes:

Figura. 8. Fuentes de financiación tradicionales.

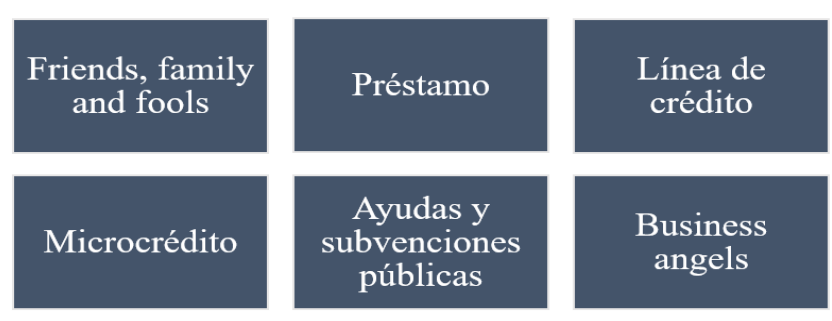

Fuente: Elaboración propia.

Estas fuentes, vistas en su conjunto, presentan las siguientes ventajas e inconvenientes para el promotor, en el momento de su utilización, siendo las ventajas las siguientes:

Entre los promotores e inversores se establece una relación estrictamente comercial, donde los roles de los participantes, los deberes y obligaciones de cada uno de los componentes de la relación, estan perfectamente definidos y se mantendrán a lo largo de la vigencia de la relación.

En el inicio de la relación entre promotor e inversor, se establecen todas especificaciones del proyecto, desde las demandas de presupuesto que surgen, a las características generales del proyecto.

- La financiación que obtiene el promotor, generalmente, la recibe en un periodo de tiempo muy reducido, en algunos casos menores de 24 horas.

- A diferencia de en los procesos de crowdfunding, en las fuentes de financiación tradicionales operan inversores profesionales, de modo que, aquel proyecto que cuenta con la aprobación de estos, 
puede considerarse como un proyecto validado de cara a otros inversores o a sus futuros consumidores.

$\mathrm{Al}$ operar inversores profesionales, pueden surgir situaciones donde estos ofrezcan al promotor no solo financiación monetaria, sino también puede ofrecerle asesoramiento y apoyo, dado que, el interés último que tienen la gran mayoría de estas fuentes tradicionales es que el inversor realiza la devolución de la financiación facilitada al promotor.

En cuanto a los inconvenientes, estos son:

- Los promotores deben de tener en cuenta de que los inversores quieren tener resultados en un período de tiempo reducido.

Los inversores buscan maximizar su inversión, es decir, elegirán aquel proyecto en donde se ofrezca una mayor rentabilidad en un periodo de tiempo menor.

En los casos de donde no se ha obtenido la financiación suficiente, el promotor no ha establecido correctamente el presupuesto o bien se producen retrasos en el plan del proyecto, se incurrirá en un mayor coste de producción que afectará en la necesidad de financiación que el promotor posee.

Cuando el promotor accede a este tipo de financiación, incurre en una deuda adicional, que es el gasto derivado de obtener la financiación, como es el caso de los prestamos, donde el promotor tiene que hacer frente al pago de comisiones.

El acceder a este tipo de financiación, el promotor, en muchas ocasiones, tiene que ceder una parte de la dirección del proyecto como condición para que el inversor te proporcione el respaldo financiero. Esta cesión, también se puede materializar en la imposición de objetivos a conseguir o bien renunciar a parte de las ganancias como pago por esta financiación.

\subsection{Comparativa entre crowdfunding social y la financiación tradicional}

Tras la exposición de las diferentes ventajas e inconvenientes, tanto del crowdfunding como de las fuentes tradicionales, se procedió a elaborar una tabla comparativa, en función de las siguientes variables de comparación:

- $\quad$ Tipo de inversor que opera (1).

- $\quad$ Fase del proyecto en la que se especializa (2).

- $\quad$ Tipo de contraprestación que el promotor tiene que ofrecer al inversor (3).

- $\quad$ Nivel de requisitos para acceder a la financiación (4).

Tabla. 1. Comparativa entre crowdfunding y fuentes de financiación tradicionales.

\begin{tabular}{|c|c|c|c|c|}
\hline $\begin{array}{l}\text { Tipo de Fuente de } \\
\text { Financiación }\end{array}$ & (1) Tipo de inversor & (2)Fase del proyecto & (3) Tipo de contraprestación & (4) Requisitos a cumplir \\
\hline Crowdfunding & $\begin{array}{l}\text { Cualquier miembro de la } \\
\text { comunidad interesada en } \\
\text { el proyecto. }\end{array}$ & $\begin{array}{l}\text { Cualquier fase } \\
\text { proyecto. }\end{array}$ & $\begin{array}{l}\text { Cuatro tipos: } \\
\text {-Devolución cantidad prestada + intereses. } \\
\text {-Productos/servicios futuros del promotor. } \\
\text {-Participaciones en los beneficios del } \\
\text { negocio. } \\
\text {-Sin contraprestación. }\end{array}$ & $\begin{array}{l}\text { Proyecto explicado con claridad, de manera que } \\
\text { sea más atractivo para la comunidad. }\end{array}$ \\
\hline $\begin{array}{l}\text { Friends, Family and } \\
\text { Fools }\end{array}$ & $\begin{array}{l}\text { Entorno cercano del } \\
\text { promotor e inversores no } \\
\text { profesionales. }\end{array}$ & $\begin{array}{l}\text { Cualquier fase del } \\
\text { proyecto, pero más } \\
\text { caracteristica en las fases } \\
\text { iniciales. }\end{array}$ & $\begin{array}{l}\text { Tres tipos: } \\
\text {-Sin contraprestación. } \\
\text {-Devolución de la cantidad prestada. } \\
\text {-Participación en el negocio. }\end{array}$ & Ninguno. \\
\hline Préstamo & Entidades financieras. & Cualquier fase. & Montante prestado + intereses. & $\begin{array}{l}\text {-Rentabilidad futura del negocio que garantice la } \\
\text { devolución del montante prestado. } \\
\text {-Garantías o avales, tanto personales como sobre } \\
\text { bienes inmuebles. }\end{array}$ \\
\hline Línea de Crédito & Entidades financieras. & Cualquier fase. & $\begin{array}{l}\text { Montante utilizado + intereses (superiores a } \\
\text { los del préstamo). }\end{array}$ & -Garantía personal. \\
\hline Microcrédito & $\begin{array}{lr}\text { Entidades } & \text { financieras, } \\
\text { asociaciones } & y \\
\text { fundaciones. } & \end{array}$ & Cualquier fase. & $\begin{array}{l}\text { Montante prestado + intereses (son bajos } \\
\text { que los de mercado). }\end{array}$ & $\begin{array}{l}\text {-Cumplimiento de los requisitos exigidos por la } \\
\text { entidad para el acceso al microcrédito. } \\
\text {-Estar dentro de la zona de actuación de los } \\
\text { programas de microcréditos existentes. }\end{array}$ \\
\hline $\begin{array}{l}\text { Ayudas y } \\
\text { subvenciones públicas }\end{array}$ & Organismos públicos. & Cualquier fase. & Ninguna. & $\begin{array}{l}\text {-Cumplir las exigencias por las cuáles puede } \\
\text { recibir la subvención o ayuda. }\end{array}$ \\
\hline Business Angels & Inversores profesionales. & $\begin{array}{l}\text { Especializada en las fases } \\
\text { iniciales. }\end{array}$ & $\begin{array}{l}\text { Alta rentabilidad futura del negocio, para } \\
\text { participar en el mismo. }\end{array}$ & $\begin{array}{l}\text {-Que el proyecto propuesto por el promotor debe } \\
\text { dar al inversor una alta rentabilidad que } \\
\text { justifique el riesgo que concurre. }\end{array}$ \\
\hline
\end{tabular}

Fuente: Elaboración propia. 


\subsection{Resultados y líneas de discusión}

Tras la elaboración y análisis de la tabla comparativa en el punto 3.3., diremos que los resultados obtenidos de esta, son los siguientes:

El crowdfunding social permite al promotor de un proyecto acceder a una mayor diversidad de inversores. Esta afirmación, coincide con lo expuesto por Sullivan (2006), Howe (2008), Belleflamme (2012), así como, Steinberg y DeMaria (2012), que definen el crowdfunding como la captación de fondos mediante el empleo de multitud, conformada tanto por una gran variedad de inversores, pudiendo estos ser personas físicas y empresas.

El crowdfunding social puede ser utilizado para cualquier etapa del proyecto. Esta afirmación, coincide con lo expuesto por Messina y Pena (2013), en su artículo «Alternativas de financiamiento a emprendedores〉, en donde se establecen las diferentes fuentes tradicionales que puede emplear el promotor atendiendo a la fase de desarrollo del proyecto.

El crowdfunding social utiliza distintas estrategias para llamar la atención de los inversores y las fuentes tradicionales se centran en enfatizar el plan de negocio. En el resultado 3, se establece que el promotor tiene que desplegar una gran variedad de conocimientos a fin de poder desarrollar una campaña de crowdfunding que le permite obtener la financiación que determino como necesaria. Esta afirmación, concuerda con lo establecido por Steinberg y DeMaria (2012), en su libro «The Crowdfunding Bible», en donde establece que el trabajo del promotor, previo a la publicación del proyecto en una plataforma es fundamental para poder atraer al mayor número de inversores, dado que, es esencial que el proyecto tenga lo siguiente:

- $\quad$ El producto o servicio futuro debe constituirse a partir de una idea sólida.

- $\quad$ Una contraprestación atractiva con el fin de atraer al inversor.

- $\quad$ Una estrategia de marketing y publicidad efectivas.

- Que la presentación del proyecto sea interesante a fin de atraer audiencia.

En cuanto a la afirmación relacionada con las fuentes tradicionales, la confederación de jóvenes empresarios (2008) coinciden con nuestra afirmación de que el inversor, en este tipo de fuentes, es de tipo profesional, dado que la financiación no procede de la comunidad, sino de organismos tanto públicos, como profesionales, como por ejemplo instituciones financiera o la administración pública.

Según la naturaleza del proyecto será mejor elegir distintas modalidades de crowdfunding y en las fuentes tradicionales no se hace ninguna diferenciación. Esta afirmación, en donde establecemos que hay una modalidad de crowdfunding perfecta para según qué proyecto, coincide con lo establecido por SajardoMoreno (2015) en su artículo «Nuevos instrumentos de financiación para el sector no lucrativo: el reto del crowdfunding social», por Gallardo (2015) en el libro «¿Qué es y cómo gestionar el crowdfunding?», así como, por Bach y Lamolla (2016) en su artículo «La financiación del emprendimiento social, ¿modelos nuevos para problemas viejos?».

Las fuentes tradicionales exigen una contraprestación, solo, de tipo monetario y el crowdfunding social permite, además, ser en especie e incluso gratuita. En esta afirmación establecemos que el promotor a la hora de captar fondos utilizando las fuentes de financiación tradicionales, debe entregar una contraprestación, de carácter monetario, como pago por estos fondos, concordando con lo establecido por la confederación de jóvenes empresarios (2008), que definen las diferentes fuentes de financiación, estableciendo que las contraprestaciones son, en su mayoría, de carácter monetario.

Por el contrario, Steinberg y DeMaria (2012), en el libro « The Crowdfunding Bible», establecen que los inversores pueden exigir al promotor, además, de una contraprestación monetaria, un cierto control sobre el proyecto, pudiendo influir en la toma de decisiones estratégicas del negocio.

Determinadas fuentes tradicionales requieren el cumplimiento de unas garantías y en el crowdfunding social no existe requisito previo. Esta afirmación, se refiere a que los promotores para poder acceder a fondos mediante el empleo de fuentes de financiación tradicionales deben de cumplir una serie de condiciones o requisitos o, bien, presentar una serie de garantías o avales, coincidiendo con lo establecido por la Confederación de Jóvenes Empresarios (2008) en su publicación «Productos Financieros y Alternativas de Financiación»». 
En las fuentes tradicionales los consejos son realizados por profesionales especializados en el sector del proyecto. Esta afirmación, coincide con lo establecido por Steinberg y DeMaria (2012), en el libro «The Crowdfunding Bible»), que nos muestra que una de las ventajas del crowdfunding es que sus inversores son más propensos a realizar sugerencias o proporcionar ideas, ya que, su objetivo no es solo obtener la contraprestación, sino que, establecen un vínculo afectivo con el proyecto y quien participa en su desarrollo y éxito.

\section{Conclusiones, limitaciones y futuras líneas de investigación}

\subsection{Conclusiones del estudio}

\subsubsection{Referentes al crowdfunding}

La utilización del crowdfunding, resalta la creatividad del género humano a la hora de buscar soluciones para problemas concretos, dado que, el aumento del uso de esta fórmula tiene una relación directa con, por un lado, el auge de internet y, por otro, la escasez de financiación para proyectos empresariales y solidarios producida en la etapa de crisis económica y financiera que sufrió nuestra sociedad en el período de 2008 a 2014, cuyos efectos todavía perduran.

Además, con la actual pandemia provocada por el COVID-19, vemos que se están estableciendo recientemente multitud de iniciativas para paliar los efectos negativos de esta situación y apoyar a aquellos sectores de la sociedad más afectados por el cierre de empresas, negocios o, bien, por la saturación de los servicios públicos. Dicho esto, consideramos que, es una fórmula que presenta una gran adaptabilidad a la naturaleza del proyecto, dado que, las diferentes modalidades existentes, poseen características concretas relacionadas con el carácter de la contraprestación que entrega el emprendedor como pago por la financiación facilitada.

Ejemplo de esto es la utilización de la modalidad de crowdfunding por donaciones para la búsqueda de financiación de proyectos solidarios, dado que, en esta modalidad, el emprendedor no tiene que realizar ningún tipo de contraprestación como pago por la financiación obtenida Prueba de ello, plataformas como "goteo.org", han establecido canales exclusivos para facilitar la promoción de proyectos solidarios relacionados con la lucha contra el COVID-19 (https://www.goteo.org/channel/coronazero).

Es un concepto que presenta un nexo común con el denominado crowdsourcing, dado que, ambos hacen uso de la multitud como medio para el desarrollo de sus actividades. Concretamente, podemos decir que el crowdfunding es la representación de un crowdsourcing específico, relacionado con la búsqueda de financiación, siendo este el origen de esta fórmula.

En la actualidad, las plataformas de crowdfunding operan en cualquier sector de actividad, empleando cualquier modalidad existente. Sin embargo, la legislación española solo regula plataformas relacionadas con el denominado crowdfunding financiero, esto es, modalidad de por préstamo e inversión, dejando el resto de plataformas, que operan bajo la modalidad de recompensa y donaciones, sin ningún tipo de regulación y a los inversores completamente desprotegidos.

\subsubsection{Referentes a las fuentes tradicionales}

Las fuentes de financiación tradicionales, son las fórmulas de financiación de proyectos más empleadas, caracterizadas por que sus inversores son, en su gran mayoría, profesionales, cuyo objetivo es la obtención de una contraprestación monetaria o una rentabilidad futura. Son figuras fuertemente controladas mediante legislación, garantizando la protección de todos los participantes.

Estas fuentes presentan la debilidad de estar condicionadas por la situación económica-financiera mundial, haciéndolas sensibles a los períodos de crisis, provocando una reducción en la concesión de fondos, llevando a que los promotores les sea muy complicado acceder a financiación para sus proyectos.

\subsection{Limitaciones encontradas}

En el transcurso de esta investigación nos hemos encontrado con dos grandes problemas, que han supuesto una dificultad a la hora de completar este estudio:

En España, no hay una normativa específica para las plataformas de crowdfunding que operan bajo la modalidad de donaciones y recompensa, ya que la normativa analizada, corresponde, en exclusiva, al denominado crowdfunding financiero, esto es, las modalidades de inversión y préstamo. 
Inexistencia de un registro oficial de plataformas de crowdfunding, que operan bajo modalidades distintas a las de préstamo e inversión, para poder determinar el número concreto de plataformas activas en España.

\subsection{Futuras líneas de investigación}

En la coyuntura actual, 2020 señalado como el año de la pandemia mundial, cuyos efectos en la economía y sociedad todavía no pueden ser cuantificados, dado que los indicadores no recogen su impacto efectivo/real, el crowdfunding puede erigirse como la alternativa perfecta a la financiación tradicional por su funcionamiento exclusivamente online, su adaptabilidad a la naturaleza del proyecto y por su prácticamente nula dependencia a los cambios económicos.

Con un IBEX35, que recoge unas caídas de más del 30\% derivado de la situación de completa incertidumbre en la que se encuentra la economía de 2020, con un incremento en la volatilidad de los activos, junto con el establecimiento de medidas de contención de la pandemia, relativas al confinamiento de la población y el cierre de negocios no esenciales. Estas condiciones negativas suponen la interrupción de las cadenas productivas mundiales, provocan a su vez, serias dificultades para el acceso por parte de las empresas a la financiación de las entidades bancarias, siendo las líneas de crédito mucho más restrictivas. Todo ello, afecta a la financiación de los negocios ${ }^{7}$ (nota al pie: si bien no se posee de la información necesaria para determinar la duración de los efectos o bien su cuantificación), pudiendo el crowdfunding de préstamo e inversión ser la herramienta perfecta para que empresas y entidades de la economía social puedan acceder a los fondos necesarios para el mantenimiento de su actividad.

Por último, cabe destacar el importante papel que está desempeñando el crowdfunding de donaciones, ya que se viene estableciendo como la principal herramienta de movilización de la sociedad, mediante el fomento en la creación de proyectos que tengan un impacto positivo en sectores afectados por la pandemia COVID-19, como, por ejemplo, iniciativas de recaudación de fondos para la adquisición material sanitario para ser empleado en hospitales, centros de salud o residencias de ancianos. Además, en base a lo anterior, resultará interesante establecer líneas de investigación relacionadas con el estudio de la utilidad del crowdfunding social en la coyuntura actual, como, por ejemplo, el papel que juega en el establecimiento de un canal de solidaridad que conecta a los ciudadanos con aquellos grupos afectados por la crisis en 2020 .

\section{Referencias bibliográficas}

Alemany, L., y Bultó, I. (2014) "Crowdfunding": nueva forma de financiación para los emprendedores. Harvard Deusto business review, No 237 , pp. 6-19.

Bach, M. y Lamolla, L. (2016) La financiación del emprendimiento social, ¿modelos nuevos para problemas viejos? Oikonomics: revista de economía, empresa y sociedad, $\mathrm{N}^{\circ}$ 6, pp. 65-72. Disponible en: http://oikonomics.uoc.edu/divulgacio/oikonomics/ca/numero06/sumari.html.

Bagheri, A., Chitsazan, H. y Ebrahimi, A. (2019) Motivaciones de crowdfunding: un enfoque en las perspectivas de los donantes. Pronóstico tecnológico y cambio social, $\mathrm{N}^{\mathrm{o}}$ 146, pp. 218-232. DOI: https://doi.org/10.1016/j.techfore.2019.05.002.

Bal, A., Weidner, K., Hanna, R. y Mills A.J. (2017) Crowdsourcing and brand control. Business Horizons, Nº 60, pp. 219-228. DOI: http://dx.doi.org/10.1016/j.bushor.2016.11.006.

Banco de España. (2020) Informe trimestral de la Economía Española. Recuperado de: https://www.bde.es/bde/es/secciones/informes/boletines/relac/Boletin_Economic/Informes trimes1/.

Belleflamme, P., Lambert, T. y Schwienbacher, A. (2012) Crowdfunding: Tapping the Right Crowd. CORE Discussion Paper $\quad N^{\circ}$. 2011/32 (Vol. 2013), pp. 3-10. https://papers.ssrn.com/sol3/papers.cfm?abstract_id=1578175.

Bellón, C. y Ruiz-Verdú, P. (2018) El crowdfunding y la financiación del emprendimiento. Papeles de Economía Española, No 157, pp. 128-150. Disponible en: https://repositorio.comillas.edu/xmlui/handle/11531/24756.

Block, J., Fisch, C., Vismara, S. y Andres, R. (2019) Private equity investment criteria: An experimental conjoint analysis of venture capital, business angels, and family offices. Journal of Corporate Finance, $\mathrm{N}^{\circ}$ 58, pp. 329-352. DOI: https://doi.org/10.1016/j.jcorpfin.2019.05.009.

Cantalapiedra, B. (2019) Crowdfunding y audiovisual de proximidad. La economía colaborativa como instrumento de desarrollo sectorial. CIRIEC-España, Revista de Economía Pública, Social y Cooperativa, No 95, pp. 257-288. DOI: https://doi.org/10.7203/CIRIEC-E.95.9095.

Si bien el Informe trimestral de la economía española, elaborado por el Banco de España, correspondiente al primer trimestre de 2020 establece que todavía los indicadores no recogen el impacto que esta pandemia, en términos cuantitativos, va a tener en la economía. Sin embargo, hay indicios (como el flujo de viajeros internacionales) que permiten pronosticar que el impacto va a ser relevante, si bien mostrando una naturaleza transitoria. 
Chaves, R., Monzón, J.L. y Zaragoza, G. (2013) La economía social: concepto, macromagnitudes y yacimiento de empleo para el Trabajo Social. Cuadernos de Trabajo Social, $\mathrm{N}^{\mathrm{o}} 26$, pp. 19-29. DOI: http://dx.doi.org/10.5209/rev_CUTS.2013.v26.n1.40269.

Confederación Española de Asociaciones de Jóvenes empresarios (2008) Productos financieros y alternativas de financiación. Álava: Aje Confederación.

Cordova, A., Dolci, J. y Gianfrate, G. (2015) The determinants of crowdfunding success: evidence from technology projects. Procedia-Social and Behavioral Sciences, N ${ }^{\circ}$ 181, pp. 115-124. DOI: https://doi.org/10.1016/j.sbspro.2015.04.872.

Echanove, J., Gómez-jordana, I. y Hierro, S. (2015) Ley 5/2015, De 27 De abril, de Fomento de la financiación empresaria. $\quad D L A \quad$ PIPER, $\mathrm{N}^{\circ} \quad 1, \quad$ pp. $6-9 . \quad$ Sitio https://www.dlapiper.com/es/spain/insights/publications/2015/05/law-for-the-development-of-business-financing/.

Estellés-Arolas, E. (2013) Relación entre el crowdsourcing y la inteligencia colectiva: el caso de los sistemas de etiquetado social. Tesis doctoral, Departamento de Organización de Empresas, Universidad Politécnica de Valencia (Valencia).

Galindo-Martín, M. Á., Méndez-Picazo, M. T. y Castaño-Martínez, M. S. (2016) Crecimiento, progreso económico y emprendimiento. Journal of innovation \& knowledge, $\mathrm{N}^{\mathrm{o}} \quad 1$, pp. 62-68. https://doi.org/10.1016/j.jik.2016.01.006.

Gallardo, E. (2015) ¿Qué es y cómo gestionar el crowdfunding? Barcelona: Editorial UOC.

García-Gutiérrez Fernández, C. (2002) La empresa de participación: características que la definen. Virtualidad y perspectivas en la sociedad de la información. CIRIEC-España, Revista de Economía Pública, Social y Cooperativa, $\mathrm{N}^{\circ} \quad 40, \quad$ pp. 99-122. Sitio https://www.researchgate.net/publication/5004197_La_empresa_de_participacion_caracteristicas_que_la_definen_ Virtualidad_y_perspectivas_en_la_sociedad_de_la_informacion.

González, A. y Ramos, J. (2018) Financiación participativa en España 2018 Informe Anual 2018. Universo Crowdfunding. p. 13. Recuperado de https://www.universocrowdfunding.com/wp-content/uploads/UC Informeanual-del-Crowdfunding-ES-EN-2018 XX92-FO2P-XZA1-32IK.pdf.

Howe, J. (2006) The rise of crowdsourcing. Wired magazine, $\mathrm{N}^{\circ}$ 14, Vol. 6, pp. 1-4.

Howe, J. (2008) Crowdsourcing. Why the power of the crowd is driving the future of business. New York: Three Rivers Press.

Jegelevičiūte, S. y Valančienè, L. (2015) Comparative analysis of the ways crowdfunding is promoted. Procedia-Social and Behavioral Sciences, No 213, pp. 268-274. DOI: https://doi.org/10.1016/j.sbspro.2015.11.536.

Kotha, R. y George, G. (2012) Friends, family, or fools: Entrepreneur experience and its implications for equity distribution and resource mobilization. Journal of business venturing, $\mathrm{N}^{\mathrm{o}}$ 27, Vol. 5, pp. 525-543. DOI: https://doi.org/10.1016/j.jbusvent.2012.02.001.

Laurell, C., Sandström, C. y Suseno, Y. (2019) Assessing the interplay between crowdfunding and sustainability in social media. Technological Forecasting and Social Change, $N^{\circ}$ 141, pp. 117-127. DOI: https://doi.org/10.1016/j.techfore.2018.07.015.

Ley 5/2015 de 27 de abril, de Fomento de la Financiación Empresarial (BOE núm. 101, de 28 de abril de 2015).

Mata, J. (2014) Las campañas de crowdfunding: su eficacia en proyectos lucrativos y causas sociales. Tesis doctoral, Facultat de Comunicació i Relacions Internacionals Blanquerna, Universitat Ramon Llull (Barcelona).

Messina, M. y Pena, J. (2013) Alternativas de financiamiento para emprendedores. En: IV Jornadas Académicas de la Facultad de Ciencias Económicas y de Administración. Universidad de la República. Disponible en: http://fcea.edu.uy/Jornadas_Academicas/2013/file/ADMINISTRACION/Alternativas\%20de\%20Financiamiento\%2 0para\%20Emprendedores.pdf.

Moreno, E. y Cazorla, L. (2016) Crowdfunding: Aspectos Legales. Navarra: Aranzadi, S.A.U.

Olarte-Pascual, C., Sierra-Murillo, Y. y Ortega, C. (2016) El papel de la comunicación en el crowdfunding: estudio de un caso de éxito cool. Revista ICONO14 Revista científica de Comunicación y Tecnologías Emergentes, № $14, \mathrm{Vol}^{\circ}$. 2, pp. 280-303. DOI: https://doi.org/10.7195/ri14.v14i2.968.

Sajardo Moreno, A., \& Pérez Sempere, S. (2018) Análisis de las plataformas de crowdfunding social en el estado español: un estudio de casos. REVESCO. Revista de Estudios Cooperativos, Primer Cuatrimestre, $\mathrm{N}^{\circ} 130$, pp. 149175. https://doi.org/10.5209/REVE.61938.

Sajardo Moreno, A., Gil Ibáñez, M., \& Pérez Sempere, S. (2017) El sector no lucrativo en el marco de la nueva era tecnológica: el desafío del Crowdfunding social en España. Análisis del caso de la asociación Alba Pérez, lucha contra el cáncer infantil. REVESCO. Revista de Estudios Cooperativos, Segundo Cuatrimestre, No 124, pp. 128-151. DOI: https://doi.org/10.5209/REVE.56132.

Sajardo-Moreno, A. (2016). Nuevos instrumentos de financiación para el sector no lucrativo: El reto del crowdfunding social. Cooperativismo y Desarrollo, № 24, Vol. 108, pp. 39-53. DOI: http://dx.doi.org/10.16925/co.v24i108.1259.

Sánchez Espada, J., Martín López, S., Bel Durán, P., \& Lejarriaga Pérez de las Vacas, G. (2018) Educación y formación en emprendimiento social: características y creación de valor social sostenible en proyectos de emprendimiento Social. REVESCO. Revista de Estudios Cooperativos, Tercer Cuatrimestre, $\mathrm{N}^{\mathrm{o}}$ 129, pp. 16-38. DOI: https://doi.org/10.5209/REVE.62492.

Son Turan, S. (2015) Financial Innovation-Crowdfunding: Friend or Foe? Procedia-Social and Behavioral Sciences, $\mathrm{N}^{\circ}$ 195, pp. 353-362. DOI: https://doi.org/10.1016/j.sbspro.2015.06.334.

Song, Y., Berger, R., Yosipof, A. y Barnes, B. (2019) Mining and investigating the factors influencing crowdfunding success. Technological Forecasting and Social Change, $\mathrm{N}^{\mathbf{0}}$ 148, pp. 119-723. DOI: https://doi.org/10.1016/j.techfore.2019.119723. 
Steinberg, S. y DeMaria, R. (2012) The Crowdfunding Bible. Disponible en: http://www.crowdfundingguides.com/The\%20Crowdfunding\%20Bible.pdf.

Torres, P. (2014) Crowdequity y crowdlending: ¿fuentes de financiación con futuro? Observatorio de Divulgación Financiera, $\mathrm{N}^{\mathrm{o}} 17$.

Tíscar, L. (2014) Crowdsourcing. Cultura compartida. En J. Celaya. (1 ${ }^{\text {a }}$ Edición). Anuario AC/E de Cultura Digital focus 2014: Uso de las nuevas tecnologías en las artes escénicas, pp.20-22. España: Acción Cultural Española.

Wang, S. y Dang, D. (2019) Incentive Mechanism for the Listing Item Task in Crowdsourcing. Information Sciences, No 512, pp. 80-95. DOI: https://doi.org/10.1016/j.ins.2019.09.067. 